\title{
Educational Disparities in Distribution of Cardiovascular Risk Factors and Quality of Care in Korean Adults: Korean National Health and Nutrition Survey IV

\author{
Jae-Moon Yoon, Ji-Hye Kim, Beom-Seok Suh, Sang Min Park*
}

Department of Family Medicine, Seoul National University Hospital, Seoul National University College of Medicine, Seoul, Korea

Background: The purpose of this study was to investigate how educational status influences cardiovascular risk factors and care of diabetes mellitus and hypertension.

Methods: From Korean National Health and Nutrition Survey IV, we obtained survey results of 6,835 men and 9,067 women more than 30 years old. We performed multivariate logistic regression to compare cardiovascular risk factors and care of hypertension and diabetes respective to educational status.

Results: There were disparities in cardiovascular risk factors by educational status. In men, impaired fasting glucose, high triglyceride, and smoking were less frequently found in the highest educated group than in the middle educated group. In women, the prevalence of abdominal obesity, impaired fasting glucose, high blood pressure, high triglyceride, and metabolic syndrome among the highest educated group were significantly lower. The proportion of those with proper physical activity in the highest educated group was found to be less than that of the middle educated group, regardless of gender. For care of diabetes mellitus and hypertension, disease recognition and treatment were lower among the lowest educated group in men, while these disparities were not shown in women. Instead, the lowest educated group in diabetic women received screening exams for eye and kidney complications less than the middle education group. In both genders, the high education group had a higher chance of receiving education about diabetes mellitus.

Conclusion: There were educational disparities in cardiovascular risk factors and care of hypertension and diabetes mellitus. The disparities were found to be different by gender.

Keywords: Education; Cardiovascular Diseases; Risk Factors; Quality of Health Care

\section{INTRODUCTION}

Received: August 31, 2011, Accepted: November 29, 2012

${ }^{*}$ Corresponding Author: Sang Min Park

Tel: +82-2-2072-3331, Fax: +82-2-766-3276

E-mail: sangmin.park.snuh@gmail.com

Korean Journal of Family Medicine

Copyright (C) 2013 The Korean Academy of Family Medicine

(a) This is an open-access article distributed under the terms of the Creative Commons Attribution Non-Commercial License (http://creativecommons.org/licenses/by-nc/3.0) which permits unrestricted noncommercial use, distribution, and reproduction in any medium, provided the original work is properly cited.
Cardiovascular diseases have been well studied, and their risk factors have been well established. In previous studies, unlike unchangeable risk factors such as family history, many risk factors including abdominal obesity, blood pressure, glucose level, lipid profile, and smoking have been demonstrated to be preventable by lifestyle modification. ${ }^{1-5)}$ However, cardiovascular diseases are still the major cause of death in Korea ${ }^{6}$ and continue to be a formidable burden on public health. In addition, hypertension 
and diabetes mellitus may be prevented from advancing to a fatal disease if properly managed.

Educational status has been known to influence the prevalence and prognosis of several diseases ${ }^{7-10)}$ and some previous studies suggests that there is association between educational status and cardiovascular risk factors. ${ }^{11,12)}$ However it has not been clear how educational status affects the management of risk factors. In our study, we assessed the relationship between educational status and cardiovascular disease. Moreover, we also compared quality of care of hypertension and diabetes mellitus to provide effective health services to vulnerable groups.

\section{METHODS}

\section{Design and Study Population}

The Korean National Health and Nutrition Survey (KNHANES) IV is a national health survey in Korea that involves population-based random sampling of 31,705 individuals in 13,800 households across 600 national districts. A stratified multistage probability sampling design (complex sampling design) was applied to the South Korean population. The survey was performed by a rolling sampling survey in 2007,2008 , and 2009. Three samples are representative of the Korean population, homogenous and independent of each other. We performed our analysis with the data collected over three years.

To report educational disparities in cardiovascular risk factors and quality of care of hypertension and diabetes mellitus, we selected proper subjects from the health examination survey for analysis. We started with cross-sectional data from 24,871 participants who had completed the health examination survey. The age was restricted to 30 and over, in order to minimize the misclassification of education status in young participants. Six thousand eight hundred and thirty-five men and 9,067 women attended the health examination and they were included to our study as first subjects. The second subjects are limited to diabetic participants to show the quality of diabetic care. Overall, 742 men and 794 women were enrolled as second subjects. Similarly, hypertensive participants were enrolled as third subjects, with a total of 2,323 men and 2,646 women. Figure 1 shows the model used to select our study population. Finally, with regard to education duration during entire lifetime, we divided subjects into groups of six years and under, seven to twelve years, and over 12 years.

\section{Outcome Variables}

Table 1 shows the definition or criteria of outcome variables in our study.

\section{1) Cardiovascular risk factors}

From KNHANES IV, we obtained data on cardiovascular risk factors. We included age, sex, smoking history, and physical activity. We also included waist circumference, blood pressure, serum glucose, and lipid profile as components of metabolic

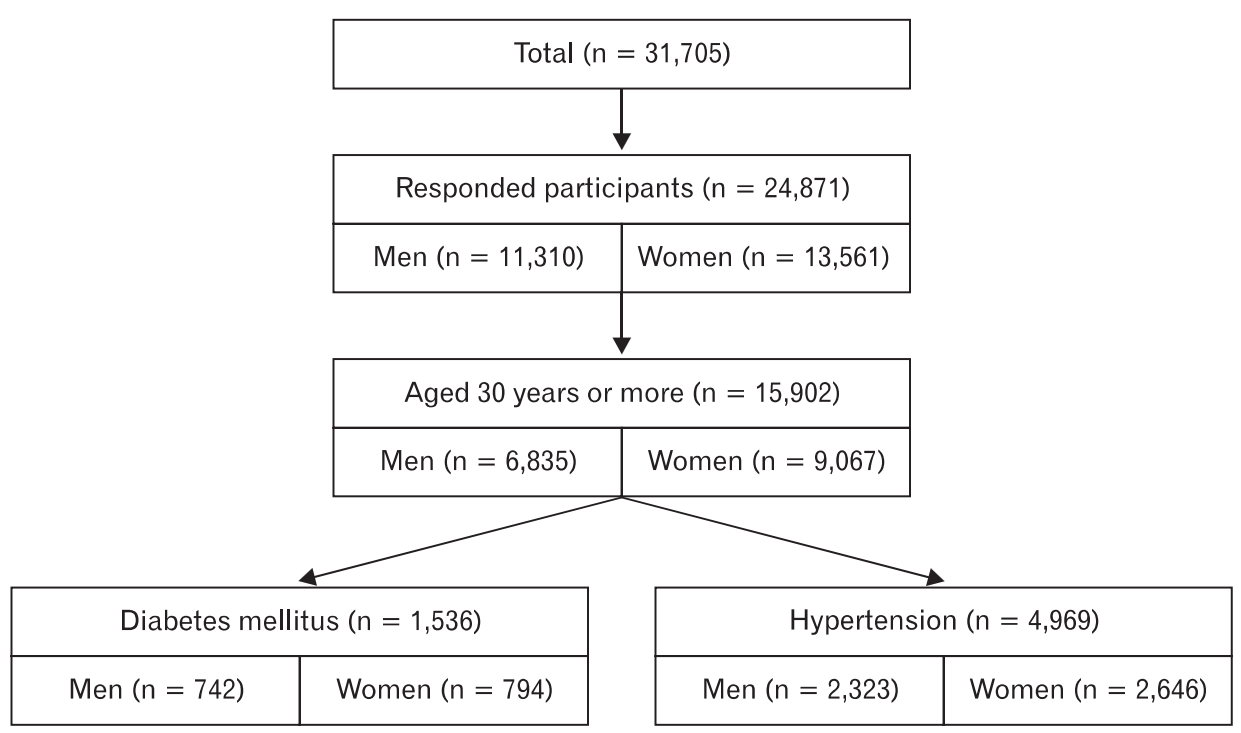

Figure 1. Study population. 
Table 1. Definition or criteria of outcome variables in this study

\begin{tabular}{|c|c|}
\hline Outcome variables & Definition or criteria \\
\hline \multicolumn{2}{|l|}{ Cardiovascular risk factors } \\
\hline Abdominal obesity & Waist circumference $>90 \mathrm{~cm}$ in men and $>85 \mathrm{~cm}$ in women according to Korean criteria \\
\hline Impaired fasting glucose & Fasting glucose level $\geq 100 \mathrm{mg} / \mathrm{dL}$ or use of antiglycemic agent(s) \\
\hline High blood pressure & $\mathrm{SBP} \geq 130 \mathrm{~mm} \mathrm{Hg}$ or $\mathrm{DBP} \geq 85 \mathrm{~mm}$ Hg or use of antihypertensive medication \\
\hline High triglycerides & Triglycerides level $\geq 150 \mathrm{mg} / \mathrm{dL}$ \\
\hline Low HDL cholesterol & HDL cholesterol level $<40 \mathrm{mg} / \mathrm{dL}$ in men and $<50 \mathrm{mg} / \mathrm{dL}$ in women \\
\hline Metabolic syndrome & $\begin{array}{l}\text { Having three or more of the followings: 1) abdominal obesity, 2) high triglycerides, 3) low } \\
\text { HDL cholesterol, 4) impaired fasting glucose or diabetes mellitus, 5) high blood pressure }\end{array}$ \\
\hline Smoking & Smoke currently or has history of smoking over 100 cigarettes during lifetime \\
\hline Exercise & Moderate intensity exercise for at least 150 minutes per week \\
\hline \multicolumn{2}{|l|}{ Care of diabetes mellitus } \\
\hline Recognition & Previous acknowledgement about self disease by doctor's diagnosis or other method \\
\hline Treatment & Receipt of prescription for glucose lowering agent \\
\hline Achievement of glycemic control & HbAlc $<7.0 \%$ among adults with known DM \\
\hline Achievement of target blood pressure & $\mathrm{SBP}<130 \mathrm{~mm} \mathrm{Hg}$ and $\mathrm{DBP}<80 \mathrm{~mm} \mathrm{Hg}$ with or without antihypertensive medication \\
\hline Achievement of target LDL cholesterol level & LDL cholesterol level < $100 \mathrm{mg} / \mathrm{dL}$ with or without lipid lowering medication \\
\hline Screening test of fundus exam & Fundus examination in the past year among adults with known DM \\
\hline Screening test of renal complication & Microproteinuria test in the past year among adults with known DM \\
\hline Exercise & Moderate intensity exercise for at least 150 minutes per week \\
\hline Education about diabetes & Received one or more times personal or group education about diabetes \\
\hline \multicolumn{2}{|l|}{ Care of hypertension } \\
\hline Recognition & Previous acknowledgement about self disease by doctor's diagnosis or other method \\
\hline Treatment & Receipt of prescription for antihypertensive medication \\
\hline Achievement of target blood pressure & $\mathrm{SBP}<140 \mathrm{~mm} \mathrm{Hg}$ and $\mathrm{DBP}<90 \mathrm{~mm} \mathrm{Hg}$ \\
\hline Exercise & Moderate intensity exercise for at least 150 minutes per week \\
\hline
\end{tabular}

SBP: systolic blood pressure, DBP: diastolic blood pressure, HDL: high density lipoprotein, HbA1c: hemoglobin A1c, DM: diabetes mellitus, LDL: low density lipoprotein.

syndrome, ${ }^{13)}$ all well known risk factors of cardiovascular disease. We did not include family history, an unchangeable risk factor.

Systolic blood pressure (SBP) and diastolic blood pressure (DBP) were measured three times. We used the mean value of the 2 nd and 3rd measurement. High blood pressure was defined as an $\mathrm{SBP} \geq 130 \mathrm{~mm} \mathrm{Hg}$ or a $\mathrm{DBP} \geq 85 \mathrm{~mm} \mathrm{Hg}$ according to the metabolic syndrome criteria. ${ }^{13)}$ Those currently being prescribed with antihypertensive agents were categorized as the high blood pressure group, regardless of their blood pressure. Impaired fasting glucose was defined as a serum glucose level of $100 \mathrm{mg} /$
$\mathrm{dL}$ or more, after 8 hours of fasting. Participants already under diabetic treatment were categorized together regardless of their glucose level. ${ }^{13)}$

From blood pressure, glucose level, waist circumference, and lipid profile, we defined metabolic syndrome. ${ }^{13)}$ Abdominal obesity was defined as waist circumference $>90 \mathrm{~cm}$ for men and $>85 \mathrm{~cm}$ for women, in reference to the Korean cutoff value. ${ }^{14)}$

Proper physical activity was defined as subjects undergoing moderate intensity exercise or walking for at least 30 minutes per day and 5 times per week from self-reported questionnaires. 
Vigorous intensity exercise for at least 20 minutes per day and 3 times per week was also classified as proper physical activity. ${ }^{15)}$ Current smoking was regarded as a risk factor if subjects smoked more than 100 cigarettes throughout their lifetime.

\section{2) Care of diabetes mellitus and hypertension}

To assess the quality of health care, we performed analyses in the diabetic and hypertensive subpopulations. In diabetic patients, we assessed recognition of disease, receipt of treatment and education about disease, disease control, management of complication, and proper exercise according to the strong recommendation of American Diabetes Association Guideline $2011 .^{16)}$ In hypertensive patients, we assessed recognition of disease, receipt of treatment, achievement of target blood pressure, and proper exercise. ${ }^{17)}$

\section{Adjustment Variables}

All data were separately analyzed by sex. We adjusted for age, the quartile of house income divided by the square-root of number of family members, residence (urban or rural), and personal health insurance. We also adjusted for thought of selfhealth status (categorized as good, ordinary, or bad) when analyzing quality of care.

\section{Statistical Analysis}

All data were analyzed using Stata ver. 12.1 (Stata Co., College Station, TX, USA). The age was analyzed using one-way analysis of variance. Socioeconomic status was compared by educational status by chi-square test. To assess cardiovascular risk factors, we used weighted-survey multivariate logistic regression (Stata command; svy: logistic) in order to reflect the characteristics of complex sampling design. However, the sample sizes of diabetes mellitus patients and hypertension patients, the highly selective populations, were too small to calculate standard error by stratified analysis. Therefore, we used conventional multivariate logistic regression without stratification and sampling weight for analysis of care of diabetes mellitus and hypertension. The reference group for logistic regression was selected as the 7 to 12 year education group, which included the majority of the population, to prevent great disparity between the lowest and highest educated group. We also analyzed the linear trend to assess the dose-response relationship.

\section{RESULTS}

\section{Socioeconomic Status}

As shown in Table 2, in both genders, educational status was significantly associated with age, house income, residence, and personal health insurance. The highly educated group consisted of younger and wealthier participants. Moreover, this group is more likely to reside in urban areas and take up personal health insurance.

\section{Cardiovascular Risk Factors}

As shown in Table 3, more educated men smoked less (P for trend $<0.01$ ) and exercised less ( $\mathrm{P}$ for trend $<0.01$ ). In men, impaired fasting glucose (odds ratio [OR], 0.81; 95\% confidence interval [CI], 0.69 to 0.95 ) and high triglyceride (OR, 0.85; 95\% CI, 0.73 to 0.99 ) were less prevalent in the highest educated groups compared to the reference. In women, education was associated with lower prevalence of all risk factors in a significant linear trend $(\mathrm{P}$ for trend $<0.01)$. It was also associated linearly with less physical activity, a protective risk factor ( $\mathrm{P}$ for trend $=$ 0.04). Additionally, the prevalence of all risk factors except low high density lipoprotein cholesterol was shown to be significantly lower in the highest educated groups compared to the reference.

\section{Care of Diabetes Mellitus}

Table 4 shows the care of diabetes mellitus and hypertension by educational status. Education is associated with recognition of diabetes in men ( $\mathrm{P}$ for trend $=0.04)$, while the effect is not apparent in women $(\mathrm{P}$ for trend $=0.72)$. Treatment of diabetes was low in the lowest educated group of men (OR, 0.62; 95\% CI, 0.41 to 0.92$)$. However, the highest educated group was associated with a lower chance of receiving treatment in women, although the statistical significance was insufficient (OR, 0.44; 95\% CI, 0.19 to 1.03$)$. Achievement of glycemic control was significantly higher in the highest educated group of women (OR, 3.16; 95\% CI, 1.30 to 7.68). Achievement of target blood pressure and low density lipoprotein cholesterol level did not show significant differences. More educated groups had a greater tendency to receive fundus examination in men ( $\mathrm{P}$ for trend < 0.01 ), whereas the lowest educated group of women received fundus examination less than the reference group (OR, 0.60; 95\% CI, 0.39 to 0.94$)$. The result of the urine exam was similar to the 

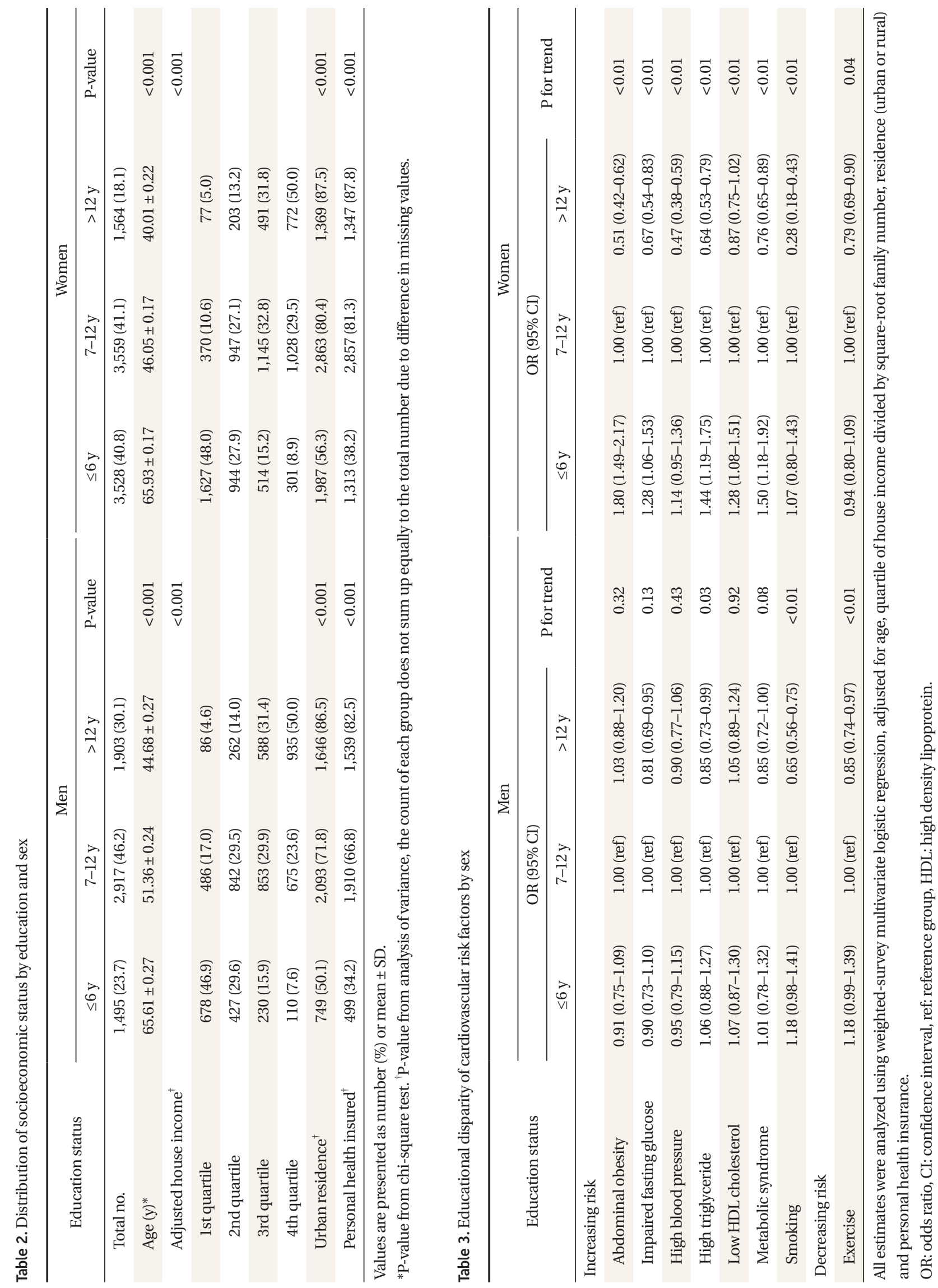


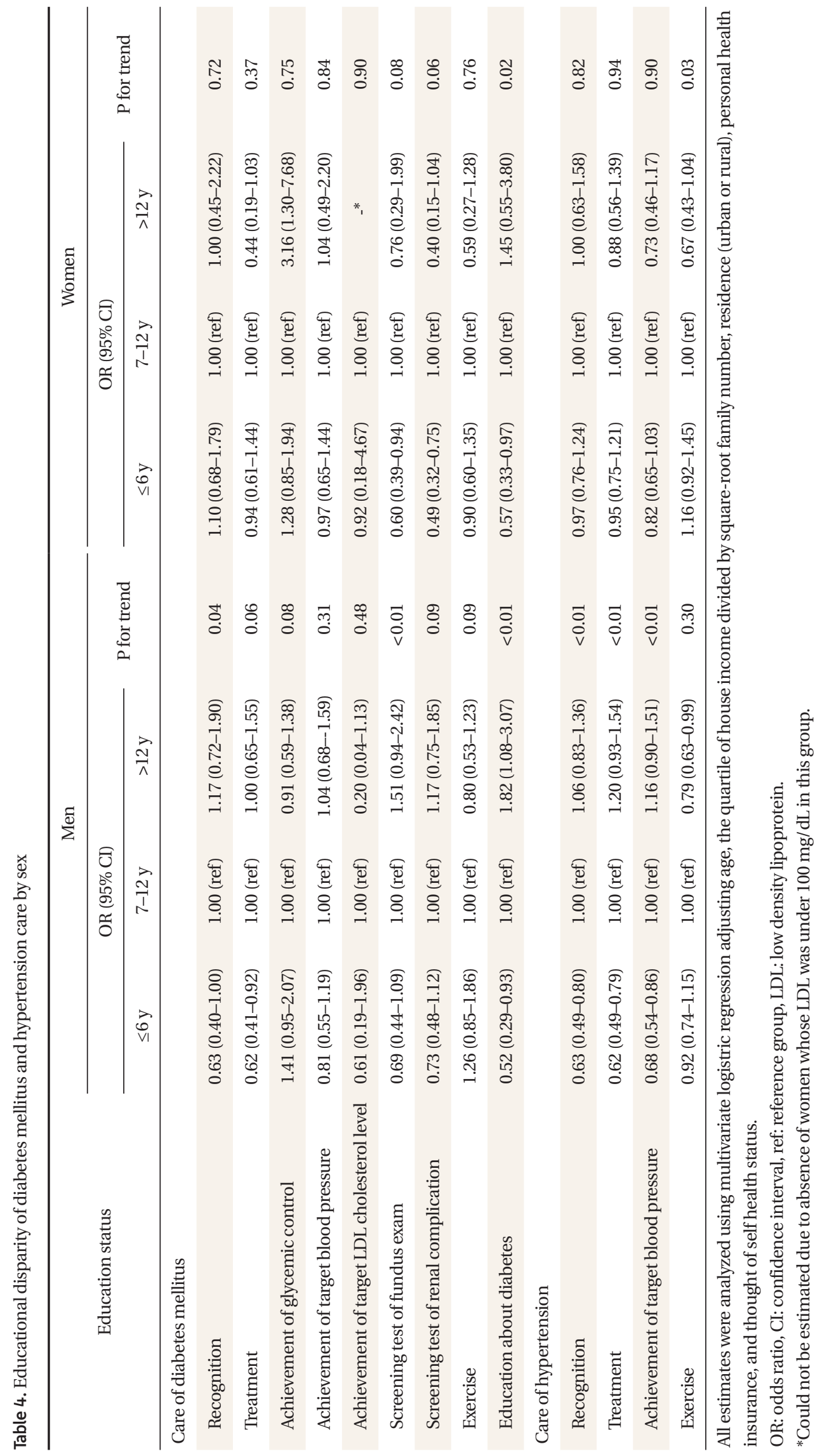


fundus exam. For physical activity there were no differences in each group, and the lowest educated groups were associated with a lower chance of receiving education about disease, regardless of gender.

\section{Care of Hypertension}

Similar to subjects with diabetes, recognition, treatment and control of hypertension in the lowest educated group were observed to be lower than the reference group in men, while no difference was shown in women. Only the highest educated group in men had significantly less proper physical activity than the reference (OR, 0.79 ; $95 \% \mathrm{CI}, 0.63$ to 0.99 ).

\section{DISCUSSION}

In our study, low educational status of women was associated with a high risk of cardiovascular disease in general. This effect was attenuated in men, but the highest educated group of men still had less risk factors compared to the reference group, specifically, impaired fasting glucose, high triglyceride levels, and smoking, which is consistent with previous studies. ${ }^{11,18)}$ However, some points need to be discussed in our study.

Firstly, educational status has a different effect on cardiovascular risk factors among men and women. We may suspect employment status as the underlying factor creating this sexual difference, because employment also is considered an important factor influencing cardiovascular risk factors. ${ }^{19)}$ According to Korean statistics, ${ }^{20)}$ educational status influences employment more in men than in women. Unfortunately, the results were not changed although adjusting employment status. From a different viewpoint, we used the same cutoff line to classify the educational status in men and women, even though the median year of education was higher in men according to our data. Consequently, the highest educated group of women corresponded to a higher degree of selection compared to that of men, which may have possibly inflated the estimates.

Secondly, there was inconsistency with a previous study, ${ }^{21)}$ in that the highest educated group was less physically active than the reference group. Our study was limited as a cross-sectional study. The causal relationship between factors cannot be determined, and reverse causality is always a possibility. For example, the highest educated group may be less motivated to exercise due to lower prevalence of other cardiovascular risk factors. Nevertheless, considering the fact that physical activity is beneficial to various medical conditions, ${ }^{17)}$ it is undeniable that physical activity in the highest educated group is insufficient.

Regarding care of diabetes mellitus, there were some significant disparities by educational status, and these findings were consistent with a previous study. ${ }^{22)}$ We observed some differences in treatment in men and achievement of glycemic control in women. However, a consistent difference was apparent in education related to disease. It is already well known that education takes an important role in diabetic care. ${ }^{23)}$ Although the guidelines recommend that all new patients receive "diabetes self-management education", only fifteen percent of participants answered that they have received diabetic education, and our results suggest that fewer among the lowest educated group receive education about disease. We could assume that the more educated group may have more opportunities to assess microvascular complications through fundus and renal screening. When we adjusted for education about diabetes, although this data was not shown, the association was no longer apparent. It suggests that the major factor to improve care is not educational status, which is an unchangeable factor of the past, but current education about disease.

Concerning exercise, the disparity between groups was minimal compared to the results of cardiovascular risk factors. We can thus assume that the major confounding factor related to exercise may be current disease status, especially diabetes mellitus.

Regarding care of hypertension, more education seemed to lead to better quality of care in men, while this tendency was attenuated in women. In our data, only 5 percent of those who had been previously diagnosed with hypertension reached the target goal of blood pressure without medication. On the contrary, the association between medication use and diabetic control appears to be weak. Our data suggest that the adherence to physician's orders may be more important in hypertension than self-awareness. ${ }^{24,25)}$ Conclusively, education may have the effect of increasing adherence to physician's orders in men, while decreasing adherence in women, even though the effect appeared to be statistically insignificant.

According to a previous study, ${ }^{26)}$ these disparities might be associated with a knowledge gap. Misconception that 
cardiovascular disease is not preventable was frequently shown in less educated people, and this could impede modification of lifestyle and receipt of proper care. High risk and poor care, interacting viciously among the lowest educated group, may be associated with increased mortality. ${ }^{27)}$ Health care and education about disease provided by educational status are expected to contribute to lower advanced disease and mortality effectively.

Conclusively, we found that there were educational disparities in cardiovascular risk factors and care of hypertension and diabetes mellitus. The disparities were found to be different by gender.

\section{CONFLICT OF INTEREST}

No potential conflict of interest relevant to this article was reported.

\section{REFERENCES}

1. Austin MA, Hokanson JE, Edwards KL. Hypertriglyceridemia as a cardiovascular risk factor. Am J Cardiol 1998;81:7B-12B.

2. Berenson GS, Srinivasan SR, Bao W, Newman WP 3rd, Tracy RE, Wattigney WA. Association between multiple cardiovascular risk factors and atherosclerosis in children and young adults. The Bogalusa Heart Study. N Engl J Med 1998; 338:1650-6.

3. Kannel WB, McGee DL. Diabetes and cardiovascular risk factors: the Framingham study. Circulation 1979;59:8-13.

4. Lindsay RS, Howard BV. Cardiovascular risk associated with the metabolic syndrome. Curr Diab Rep 2004;4:63-8.

5. Wilson PW. Established risk factors and coronary artery disease: the Framingham Study. Am J Hypertens 1994;7(7 Pt 2):7S-12S.

6. Statistics Korea. Life table by the cause of death in 2009 [Internet]. Daejeon: Statistics Korea [cited 2011 Aug 11]. Available from: http://kosis.kr/abroad/abroad_01List.jsp.

7. DeSantis C, Jemal A, Ward E. Disparities in breast cancer prognostic factors by race, insurance status, and education. Cancer Causes Control 2010;21:1445-50.

8. Icli F, Akbulut H, Yalcin B, Ozdemir F, Isıkdogan A, Hayran
$\mathrm{M}$, et al. Education, economic status and other risk factors in gastric cancer: "a case-control study of Turkish Oncology Group”. Med Oncol 2011;28:112-20.

9. Kurzinger ML, Pagnier J, Kahn JG, Hampshire R, Wakabi T, Dye TD. Education status among orphans and non-orphans in communities affected by AIDS in Tanzania and Burkina Faso. AIDS Care 2008;20:726-32.

10. Tavani A, Fioretti F, Franceschi S, Gallus S, Negri E, Montella $\mathrm{M}$, et al. Education, socioeconomic status and risk of cancer of the colon and rectum. Int J Epidemiol 1999;28:380-5.

11. Gupta R, Kaul V, Agrawal A, Guptha S, Gupta VP. Cardiovascular risk according to educational status in India. Prev Med 2010;51:408-11.

12. Reddy KS, Prabhakaran D, Jeemon P, Thankappan KR, Joshi P, Chaturvedi V, et al. Educational status and cardiovascular risk profile in Indians. Proc Natl Acad Sci U S A 2007;104:162638.

13. Grundy SM, Brewer HB Jr, Cleeman JI, Smith SC Jr, Lenfant C; National Heart, Lung, and Blood Institute, et al. Definition of metabolic syndrome: report of the National Heart, Lung, and Blood Institute/American Heart Association conference on scientific issues related to definition. Arterioscler Thromb Vasc Biol 2004;24:e13-8.

14. Lee SY, Park HS, Kim DJ, Han JH, Kim SM, Cho GJ, et al. Appropriate waist circumference cutoff points for central obesity in Korean adults. Diabetes Res Clin Pract 2007;75:7280.

15. US Department of Health and Human Services. 2008 Physical activity guidelines for Americans [Internet]. Washington (DC): US Department of Health and Human Services; 2008 [cited 2011 Aug 11]. Available from: http:// www.health.gov/paguidelines/guidelines/default.aspx.

16. American Diabetes Association. Standards of medical care in diabetes--2011. Diabetes Care 2011;34 Suppl 1:S11-61.

17. Joint National Committee on Prevention, Detection, Evaluation, and Treatment of High Blood Pressure; National Institutes of Health; National Heart, Lung, and Blood Institute; National High Blood Pressure Education Program; National Center for Biotechnology Information. The seventh report of the Joint National Committee on Prevention, Detection, Evaluation, and Treatment of High Blood Pressure: NIH publication, no. 04-5230. Bethesda: National 
Heart, Lung, and Blood Institute; 2004.

18. Kaplan GA, Keil JE. Socioeconomic factors and cardiovascular disease: a review of the literature. Circulation 1993;88(4 Pt 1): 1973-98.

19. Popham F, Mitchell R. Relation of employment status to socioeconomic position and physical activity types. Prev Med 2007;45:182-8.

20. Statistics Korea. Economically active population by educational status from 2010 July to 2011 July [Internet]. Daejeon: Statistics Korea [cited 2011 Aug 11]. Available from: http://kosis.kr/abroad/abroad_01List.jsp.

21. Kershaw KN, Mezuk B, Abdou CM, Rafferty JA, Jackson JS. Socioeconomic position, health behaviors, and C-reactive protein: a moderated-mediation analysis. Health Psychol 2010;29:307-16.

22. Bosma H, Lamers F, Jonkers CC, van Eijk JT. Disparities by education level in outcomes of a self-management intervention: the DELTA trial in The Netherlands. Psychiatr Serv 2011;62:793-5.
23. Ayyagari P, Grossman D, Sloan F. Education and health: evidence on adults with diabetes. Int J Health Care Finance Econ 2011;11:35-54.

24. Redon J, Erdine S, Bohm M, Ferri C, Kolloch R, Kreutz R, et al. Physician attitudes to blood pressure control: findings from the Supporting Hypertension Awareness and Research Europe-wide survey. J Hypertens 2011;29:1633-40.

25. Turchin A, Conlin PR. The doctor needs to see you now: accelerating the care of patients with uncontrolled hypertension. Expert Rev Cardiovasc Ther 2010;8:1501-3.

26. Kandula NR, Tirodkar MA, Lauderdale DS, Khurana NR, Makoul G, Baker DW. Knowledge gaps and misconceptions about coronary heart disease among U.S. South Asians. Am J Prev Med 2010;38:439-42.

27. Malyutina S, Bobak M, Simonova G, Gafarov V, Nikitin Y, Marmot M. Education, marital status, and total and cardiovascular mortality in Novosibirsk, Russia: a prospective cohort study. Ann Epidemiol 2004;14:244-9. 